\title{
DELTA PHASE IN INCONEL 718: \\ MECHANICAL PROPERTIES AND FORGING PROCESS REQUIREMENTS
}

\author{
Y.Desvallées, M.Bouzidi, F.Bois and N.Beaude \\ SNECMA, Materials and Processes Department \\ BP 81 - 91003 EVRY - FRANCE
}

\begin{abstract}
To fullfill the Inconel 718 requirements, it is necessary to understand the influence of delta phase on microstructure evolution and on mechanical resistance. Therefore three comprehensive studies have been carried out:

- a validated delta phase characterization methodology,

- a review of the main effects of delta phase (content/morphology) on mechanical properties: delta content affects yield strength and both content and morphology affect creep rupture properties, - a validated delta solutionning model predicting content evolution and a study pointing out the parameters of delta particles pinning the grain boundary.
\end{abstract}




\section{Background}

Over the last few years, the LCF resistance of Inconel 718 critical parts in civil engines (i.e. turbine and compressor disks) under a hot environment (up to $650^{\circ} \mathrm{C}$ ) has been significantly increased.

Therefore fine grains grades 718 have been developed (namely HS and $D A$ ). The main problem for fullfilling DA 718 requirements is to keep delta phase during forging process and to minimize its content in the final part.

To achieve that challenge, it is necessary to understand the influence of delta phase on microstructure evolutions and on mechanical resistance. Therefore three comprehensive studies have been successively carried out: microstructural characterization of delta phase through an original method using Image Analysis; synthesis of SNECMA's knowledge over the past twenty years about the influence of delta phase on mechanical properties of industrial parts; quantification of the evolution kinetics of delta phase and recrystallization.

\section{Microstructural investigations}

It is possible to establish an accurate correlation between delta phase microstructural parameters such as delta content, and useful parameters for the forger as far as sensitive enough delta phase evaluation methodology can be used.

Two approaches have been successively carried out to determine the individual geometry of delta particles and the delta content.

In order to reach the typical 3D morphology of the delta particles, deep etching of the gamma matrix or even delta phase extraction by an electrolytic process are performed. Then actual sizes of the particles are measured on a couple of scanning electron microscope (SEM) stereographic views.

Quantitative measurements of delta content are performed by use of image analysis. A classical microstructural analysis device connected to a SEM and providing images with a $512 * 512$ pixels definition on 256 grey levels is used. The main difficulty for computerizing is to accurately digitalize the microstructure. The sample is prepared with stringent precautions: polishing followed by etching that attacks uniformly the gamma solid solution which is recommended for image analysis [1]. Image acquisition needs both secondary and backscattered electrons and the image quality is improved by means of processing operations.

\section{General image processing:}

The choice of magnification is such that a compromise must be found between the size of the smallest delta particles we want to keep and the number of particles contained in an investigation field. This parameter can be adjusted by multiplying the number of fields.

Afterwards, the magnification value is taken into account by the pixel size 0.0476 micron. The right number of fields is determined by the stabilization of the average of delta content ( $f i g$ 1). In order to enhance the contrast between the delta phase and the matrix gamma in the background, a Laplacian filter is first applied. In the corresponding binary 
image, only particles with the largest size over 5 pixels are considered. The percentage of delta phase is determined for such magnification and averaged after 60 fields.

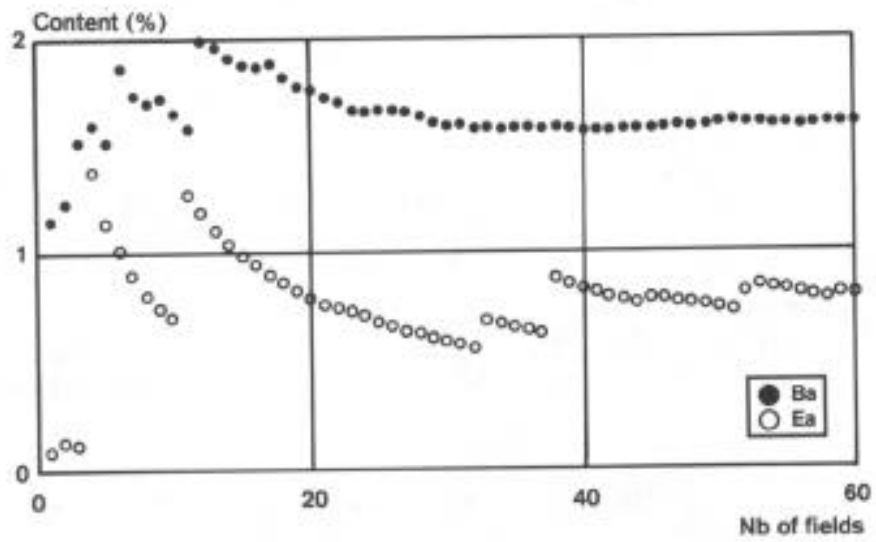

Fig. 1: Determination of the right number of investigation fields

Microstructural results:

Up to now, the forger used to distinguish two kinds of morphologies of delta particles on polished and etched samples: spheroids and needles. After partial or total extraction, it seems that the morphologies of the so-called needles are platelike, where the three dimensions are all different. The third one is particularly small with a thickness around 0.10 micron ( $\mathrm{fig} \mathrm{2)}$ ).

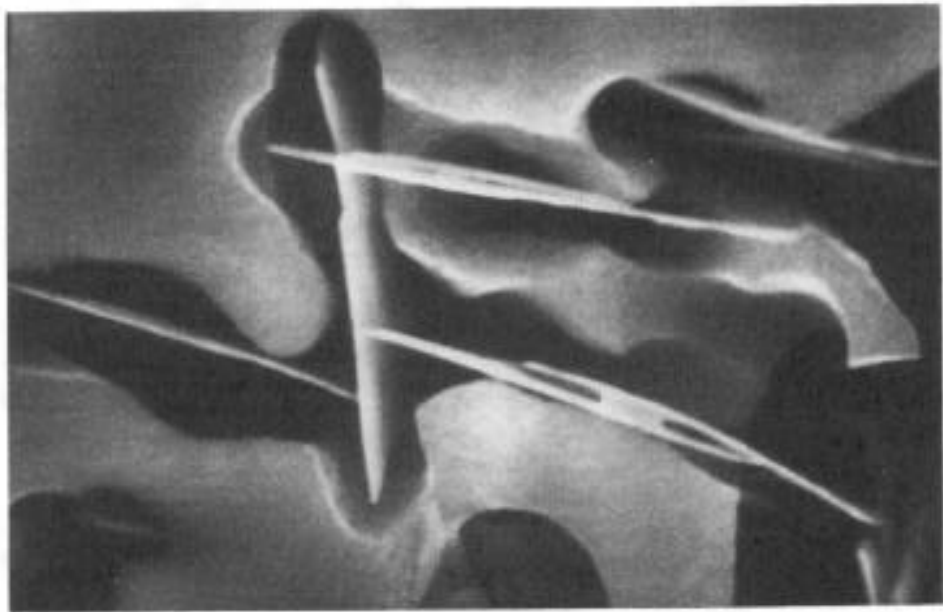

Fig. 2: 3D morphology of delta particles

Concerning the so-called spheroidal structure, it appears platelike with about the same thickness ( 0.1 to 0.2 micron) and the two other dimensions smaller than the platelike morphology.

Image analysis as a delta content measurement method has been a posteriori validated thanks to the consistency of the 
solution study results. Delta content measurement procedure has also been applied for several test specimens and especially for two types of delta microstructures used for the mechanical properties study.

\section{Mechanical properties}

\section{Experimental procedure:}

To understand the influence of delta phase, we have to know for each mechanical property, which are the influent parameters and $\mathrm{fix}$ them. Here we are just interested in two amounts of delta phase:

- type "A": in which the delta phase is present only at the grain boundaries, typically it is the standard 718 (fig.3a)

- type "B": the delta phase is in great quantity present everywhere in the structure (inter/transgranular) ( $\mathrm{fig} .3 \mathrm{~b})$

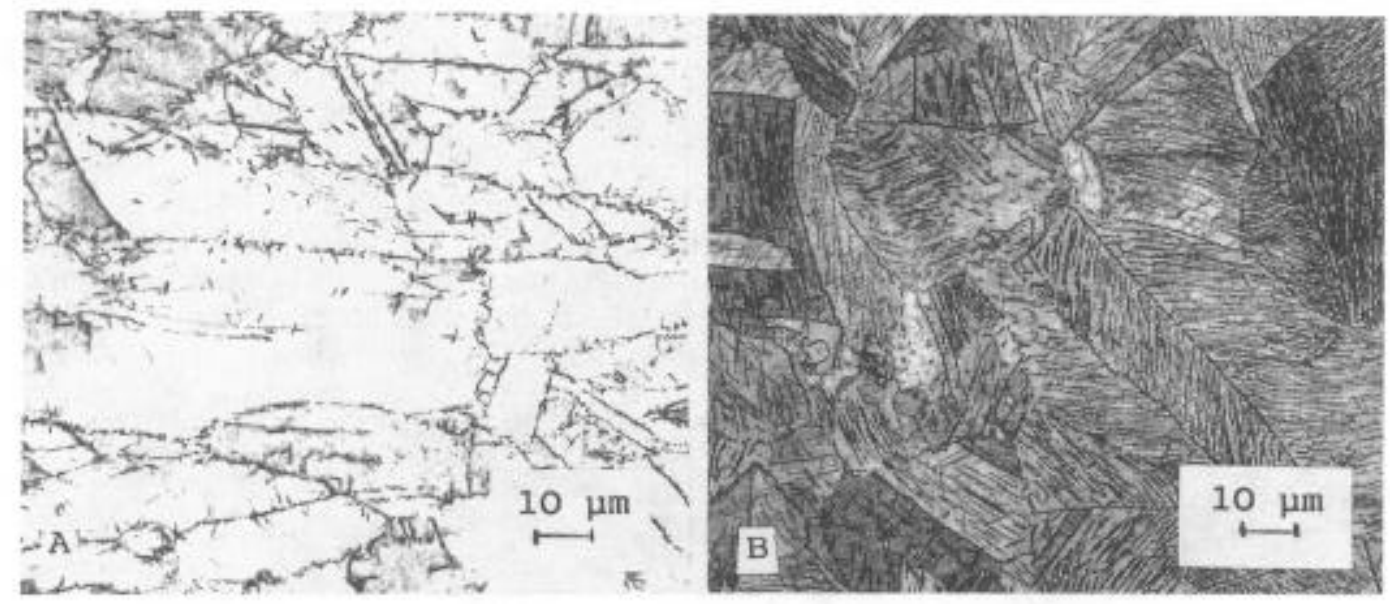

Fig. $3 a$

Fig. 3b

Fig. 3: Typical delta optical microstructures: 3a: type "A" $(\delta=38)$ and $3 \mathrm{~b}$ : type "B" $(\delta=10 \%)$

Tensile:

The influent parameter in tensile are -residual cold work

-gamma", the hardening phase

-grain size, following the Hall-Petch law

In order to explain the influence of delta phase, we fixed the parameters (except gamma"), choosing the samples A and B in the same part but located in different areas which have the same grain size $4 \mathrm{ASTM}$ and similar estimated cold work.

The results of room temperature and $1022^{\circ} \mathrm{F}\left(550^{\circ} \mathrm{C}\right)$ tensile test for both types are shown in fig.4. The type "A" results are mixed with a generic curve of standard 718 , its own results are centered around the mean curve in ultimate tensile strength (UTS) and yield stress (YS). The UTS of type "B" is as good as the standard 718, but type "B" shows a 10 s fall compared to type "A" in YS and also a better elongation. 


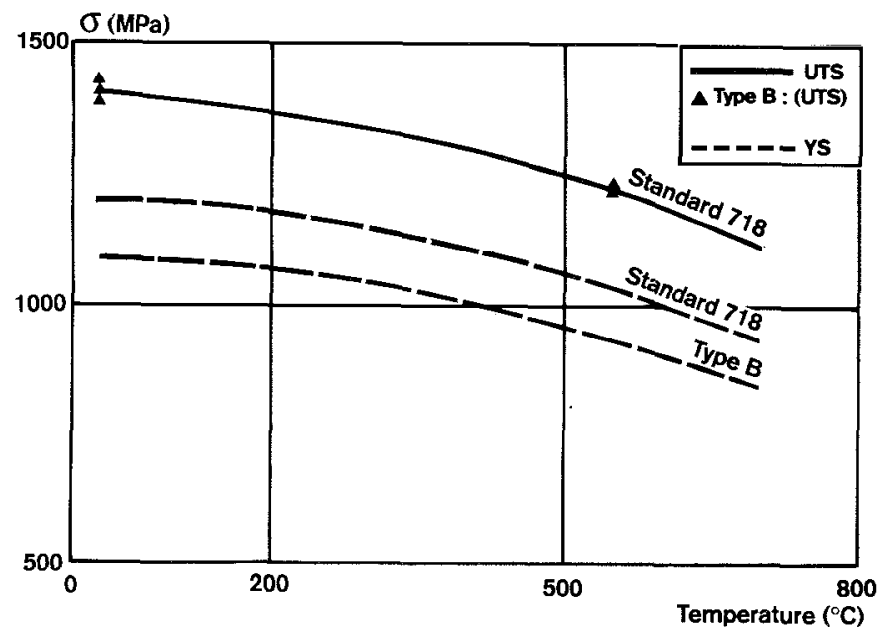

Fig. 4: Tensile results

\section{Creep:}

The influent parameters in creep are:

- grain size: bigger is the grain, higher is the creep rupture life

- cold work: it reduces the time spent during the first step (creep dislocation)

- Mg, S: they modify the carbide and delta phase shape (from platelike to spheroid), and increase the secondary creep strain rate $[2,3]$

- delta phase: at $1100^{\circ} \mathrm{F}\left(593^{\circ} \mathrm{C}\right)$ J.R. Groh [4] shows that, as delta phase amount increases, to.2\% decreases. Nevertheless, in creep elongation, according to our studies, delta phase is not a major parameter.

In creep rupture, Y.Honnorat [5] shows that at $1200^{\circ} \mathrm{F}$ $\left(650^{\circ} \mathrm{C}\right)$ when delta varies from standard to type "B" (other parameters fixed), the creep rupture life is weaker ( $\mathrm{fig} .5$ ). The type "B" are centered around the M-3s curve of the standard material. Additionnal heattreatment at $1010^{\circ} \mathrm{C}$ was made on the part and we observed that delta phase is solutionned and that its shape is only spheroidal, the grain size is bigger, the creep rupture life is then centered on the mean curve.

In conclusion, delta phase has a detrimental effect on the creep rupture life in amount and in shape, the platelike are more armfull than the spheroidal because they are a preferential site for cavity growth.

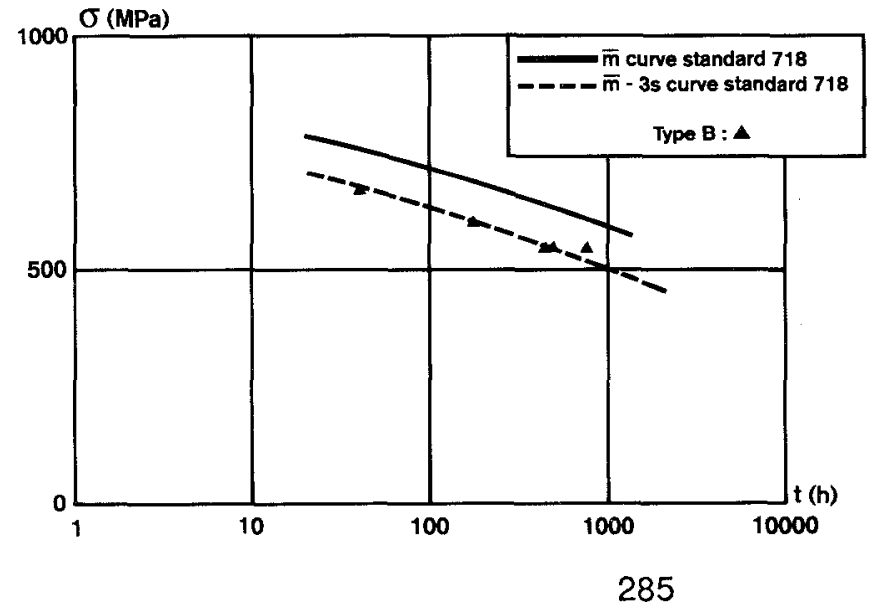

Fig, 5: Creep results 


\section{Low Cycle Fatigue:}

The parameter which controls the LCF is the grain size, cold work being a second order parameter. Many tests have been performed at SNECMA on type "B" delta phase structure without finding any direct effect on life time in LCF, at every test temperature (fig.6). Delta phase has an indirect effect on $L C F$, when it precipitates in the cooling before the forging, it can inhibit the recrystallization, and then we obtain a duplex microstructure with the big unrecristallized grains and full of delta phase. Because of the relationship between grain size and LCF, resulting in a duplex structure leads to a smaller time of failure than a fine homogeneous structure. By this way, delta phase can affect LCF.

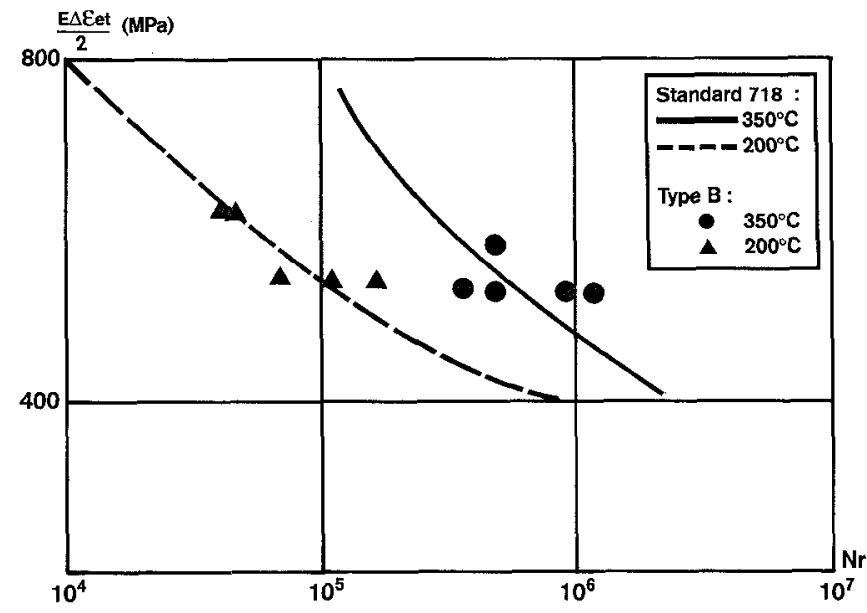

Fig. 6: Low Cycle Fatigue results

Forger's main concerns with Inconel 718

To succeed refining the grain from ASTM 4 to 10 at least, the forger has to perform a minimum of two deformation steps inducing each complete dynamic+metadynamic recrystallization, the first having to reach ASTM 8. But to keep this intermediate grain during the second heat, the last operation is to be sub-delta solvus.

Therefore, the first issue of the forger is to keep delta phase to pin the grain boundaries during the heating step and the forging step (effect of the adiabatic heating). Two questions must then be answered:

*which parameters among content/morphology or spacial distribution are the most significant to control grain growth?

$*_{\text {which }}$ parameters among temperature/time/local chemie control the evolution kinetics of delta phase?

The second issue is to avoid heavy delta-phase precipitation during the heating and cooling steps around $900^{\circ} \mathrm{C}$ and due to excessive cold-work. This issue, mainly linked to the thickness of the dead zone (cold-worked unrecrystallized region), can be solved several ways. This issue is not developed here. 
Characterization of the equilibrium state:

Content: neglecting the influence of $\mathrm{Ti}$ and $\mathrm{Al}$ on delta-phase formation and neglecting the $\mathrm{Nb}$ trapped in gamma" or $\mathrm{NbC}$, let us consider the chemical equilibrium:

$3 \mathrm{Ni}+\mathrm{Nb} \longleftrightarrow \mathrm{Ni}_{3} \mathrm{Nb}(\delta)$

The rate constant $\mathrm{k}$ for this reaction is given by:

$$
K=\frac{a(\delta)}{a(N i)^{3} * a(\mathrm{Nb})}
$$

where $a(x)$ is the activity of element $x$.

Assuming ideal behaviour, and writing the equilibrium law;

$-\mathrm{R} \cdot \mathrm{T} \cdot \ln (\mathrm{K})=\delta \mathrm{G}^{\circ}$,

where $\delta G^{\circ}$ is the free enthalpy of formation of delta phase from solid solution,

we obtain the equation giving delta-phase content vs temperature, and also the delta-solvus for any chemical composition,

Morphology: we have experimented that the stable morphology is mainly dependent on temperature.

For heat treatments over $1010^{\circ} \mathrm{C}$, the stable morphology is spheroid. Under $930^{\circ} \mathrm{C}$, it is platelike. According to Sundararaman \& al [6] at $950^{\circ} \mathrm{C}$ and to our studies at $985^{\circ} \mathrm{C}$, both spheroidal and platelike morphologies are observed.

\section{Solution kinetics:}

Solutionning specimens initially containing delta-phase leads with a varying kinetics to both chemical and morphology evolutions. We have therefore to appreciate and compare the two kinetics. At a temperature over the delta solvus, the equilibrium state corresponds to zero delta phase. The question is then to know what is to happen first: solution or spheroidization.

Material history: the Inconel 718 billet in this chapter is VIM/VAR produced and $\phi 250 \mathrm{~mm}$ delivered. The billet has been forged twice in impression dies and the specimens have been machined in the test ring of the as-forged part. We have then a good reliability concerning the homogeneity among the specimens. The chemistry controlled on the test-ring is:

$.029 \mathrm{C}, 18.45 \mathrm{Cr}, 3.05 \mathrm{Mo}, 0.18 \mathrm{Co}, 0.58 \mathrm{Al}, 1.03 \mathrm{Ti}, 18.54$ Fe, $5.30 \mathrm{Nb}$. The equilibrium solution curve for this chemistry is displayed in fig 7. The reference microstructure is shown in $\mathrm{fig.8.}$ 


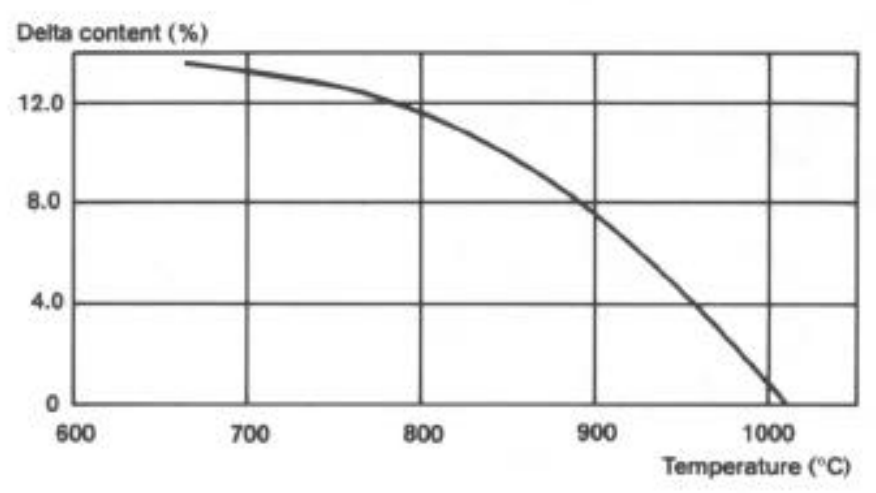

Fig. 7: Equilibrium delta content vs temperature

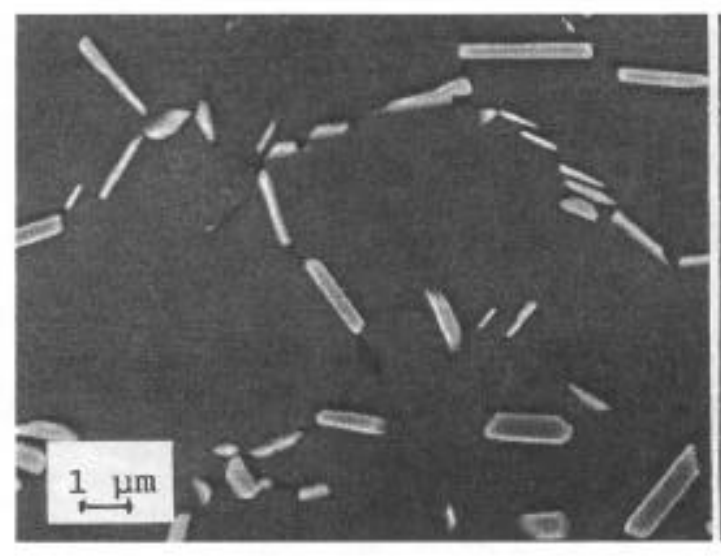

Fig. 8: Reference microstructure for solution kinetics study

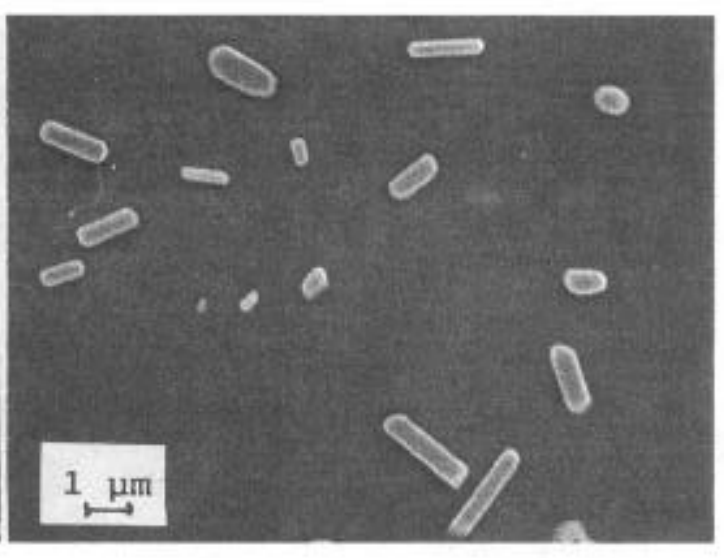

Fig. 10: Sample P $985^{\circ} \mathrm{C}-6 \mathrm{~h}$ treated

Solution test matrix: we have then heat treated samples (40mm $x 10 \mathrm{~mm} \times 10 \mathrm{~mm}$ ) in a quick response furnace following this solution test-matrix:

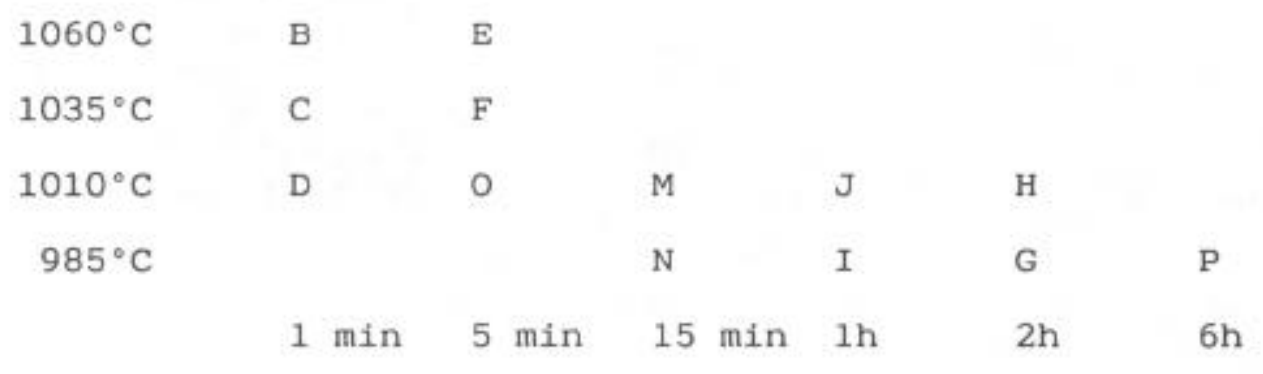

Results: the delta contents, measured with the described image analysis method, are shown on fig. 9. Initial amount is estimated at $5.5 \%+/-0.5 \%$. Fraction decreases with increasing temperature and solution time. 
The time to reach the chemical equilibrium at the grain scale is very dependent on temperature and probably less on initial structure. We observed that it is about 10 minutes at $1060^{\circ} \mathrm{C}$ and above 6 hours at $985^{\circ} \mathrm{C}$.

The fraction at $1010^{\circ} \mathrm{C}$ decreases to $0 \%$ tangentially and at $985^{\circ} \mathrm{C}$ decreases to about 2.5\% (see sample $\mathrm{P}$ in fig.10): the delta solvus is then very near to $1010^{\circ} \mathrm{C}$, and these results are consistent with the solution curve.

It has been established a model predicting delta phase content evolution for any temperature/time cycle over the delta solvus. Good fitting is shown on fig.9. Further studies will try and estimate the influence of delta particles' thickness on solution times.

It seems that solution and morphology evolution kinetics are strongly coupled because diffusion of $\mathrm{Nb}$ in the matrix is governing both solution and morphology evolutions.

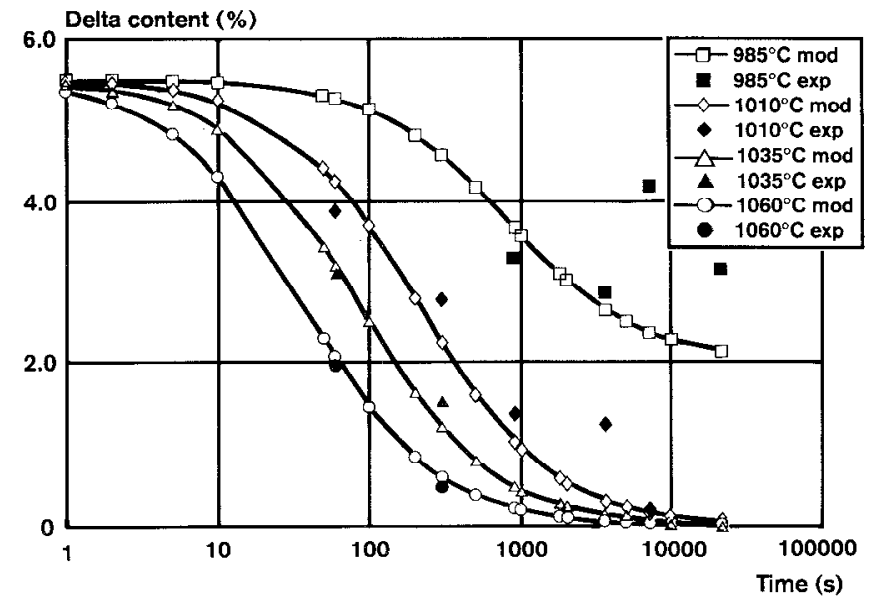

Fig. 9: Evolution of delta content: experimental points and predictive curves

Grain growth:

Grain size determination is made by ASTM E112 and the grain size results are shown in fig. 11. Initial grain size is ASTM 12 .

It appears that delta phase may whether inhibit, lower or have no effect on the kinetics of grain growth [8]. We try thereafter to precise delta phase effect on grain growth, adding our specific experimental results:

Effect of fraction: no grain growth occurs over a delta content of $4 \%$, which is comparable to results of A.W.Dix \& al [7]. We also observe at $985^{\circ} \mathrm{C}$ that with around $2.5 \%$ of delta (which is the equilibrium content at this temperature), the static grain growth kinetics drastically lowers but isn't inhibited. Under $0.5 \%$, it appears that delta phase has no effect. 
Effect of morphology: it has been noticed that platelike particles are more efficient to prevent grain growth than spheroids. This can be explained, assuming that particles border grain boundaries, considering that it is the surface of particle that prevent grain growth (see fig. 12).

Effect of spacial distribution: the two forging steps have each generated a specific grain structure. Between the two deformation steps, delta has precipitated on the former grain structure, which size has been estimated about ASTM 8. During high temperature hold time, solutionning of delta-phase takes place successively for these two networks; this mecanism is responsible for the changes of slopes in fig.11.

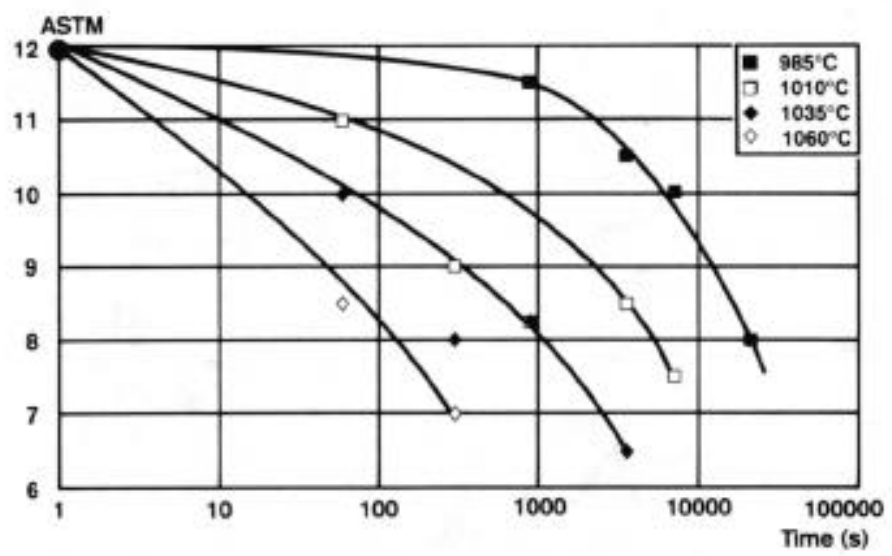

Fig. 11: Grain size evolution

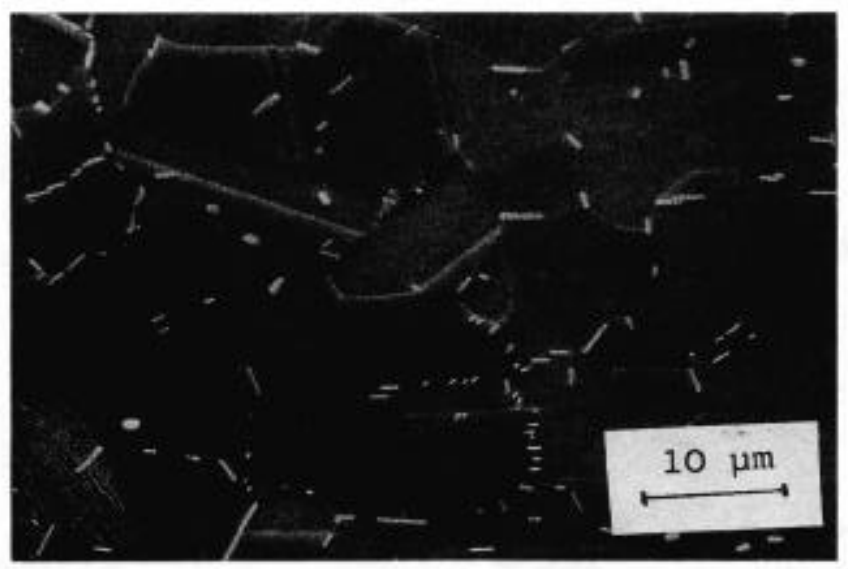

Fig. 12: Example of a delta morphology that prevents grain growth 


\section{Conclusion}

A delta phase characterization methodology has been developed. The individual 3D morphology of delta particles are platelike with a thickness around $0.1 \mu \mathrm{m}$ on both spheroid and platelike structures. Quantitative results such as delta content have been used to validate an evolution kinetics model.

The influence of delta phase on the mechanical properties especially on tensile, creep rupture, LCF has been studied. It has been shown that the presence of delta phase has:

- a detrimental effect on yield strength (-108 compared to standard 718) because it induces a lack of gamma" but no effect on ultimate tensile strength has been found

- a faint influence on time to $0.2 \%$ creep elongation but is an important parameter for creep rupture resistance especially in well oriented platelike structures,

- no direct effect on LCF but it can indirectly affect the grain structure generated by forge operations.

The effect of thermomechanical processing (TMP) on delta phase is well described through the modelings of delta content equilibrium curve and of TTT solution diagram. Yet our experiments show that delta content is not the only parameter that controls grain growth and that delta precipitate's morphology and spacial distribution should be examined.

\section{References}

1. S.T.Wlodek, Microstructural Science, vol 18, Editors T.A.Place and al., 1990, pp 407-429

2. X.Xie and al, Superalloys 1988, AIME (1992) pp 635-642

3. X.Xie and al, Superalloys 1984, pp 611-620

4 J.R.Groh, Final report GE

5 Y.Honnorat, SNECMA internal diffusion, 1985

6 M. Sundararaman \& al, Metallurgical Transaction A, volume 19 A - March 1988 pp 453-465

7 A.W.Dix, J.M.Hyzak and R.P.Singh, Superalloys 1992 pp 22-32

8 J.F. Radavich \& W.H.Couts Jr, Superalloys 1984 pp497-507 